\title{
First-order nonthermal phase transition after preheating
}

\author{
S. Khlebnikov, ${ }^{1}$ L. Kofman, ${ }^{2}$ A. Linde, ${ }^{3}$ and I. Tkachev ${ }^{1,4}$ \\ ${ }^{1}$ Department of Physics, Purdue University, West Lafayette, IN 47907, USA \\ ${ }^{2}$ Institute for Astronomy, University of Hawaii, 2680 Woodlawn Dr., Honolulu, HI 96822, USA \\ ${ }^{3}$ Department of Physics, Stanford University, Stanford, CA 94305-4060, USA \\ ${ }^{4}$ Institute for Nuclear Research of the Academy of Sciences of Russia, Moscow 117312, Russia
}

(April 1998)

\begin{abstract}
During preheating after inflation, parametric resonance rapidly generates very large fluctuations of scalar fields. In models where the inflaton field $\phi$ oscillates in a double-well potential and interacts with another scalar field $X$, fluctuations of $X$ can keep the $\phi \rightarrow-\phi$ symmetry temporarily restored. If the coupling of $\phi$ to $X$ is much stronger than the inflaton self-coupling, the subsequent symmetry breaking is a first-order phase transition. We demonstrate the existence of this nonthermal phase transition with lattice simulations of the full nonlinear dynamics of the interacting fields. In particular, we observe nucleation of an expanding bubble.
\end{abstract}

Cosmological phase transitions are one of the central topics of modern cosmology [1. Recently, this theory was supplemented by the possibility of nonthermal cosmological phase transitions [2], i.e. phase transitions driven by fluctuations produced so rapidly that they did not have time to thermalize. Large nonthermal fluctuations naturally occur in inflationary models during preheating [3].

Fluctuations of Bose fields generated by the parametric resonance during preheating have large occupation numbers and can be considered as interacting classical waves, which allows to study the dynamics of fluctuations during and after preheating using lattice simulations 朋. Numerical calculations, as well as analytical estimates 44 11] have shown that the maximal values achieved by fluctuations can be large enough to cause cosmologically interesting phase transitions. Nevertheless, until now there was no direct demonstration of the existence of such phase transitions; several groups which studied this issue numerically [12] either concentrated on models where phase transitions cannot occur, or neglected essential feedback effects such as rescattering of created particles.

We have performed a number of lattice simulations of nonthermal phase transitions, which demonstrated formation of various types of topological defects [13]. In this Letter we report results that prove that nonthermal phase transitions may take place after preheating even on the scale as large as the GUT scale $\sim 10^{16} \mathrm{GeV}$. The phase transition that we have found is first-order, which may have particularly important cosmological implications. First-order phase transitions have a very clear signature: they proceed through nucleation and subsequent expansion of a bubble of the new phase inside the old phase. In our opinion, the presence of this distinctive signature eliminates all doubts about the possibility of nonthermal phase transitions in the class of theories under investigation.

As a prototype we will use the model
$L=\frac{1}{2}\left(\partial_{\mu} \phi\right)^{2}+\frac{1}{2}\left(\partial_{\mu} X\right)^{2}-\frac{\lambda}{4}\left(\phi^{2}-v^{2}\right)^{2}-\frac{g^{2}}{2} \phi^{2} X^{2}$.

The inflaton scalar field $\phi$ has a double-well potential and interacts with an $N$-component scalar field $X ; X^{2}=$ $\sum_{i=1}^{N} X_{i}^{2}$. For simplicity, the field $X$ is taken massless and without self-interaction. The fields couple minimally to gravity in a FRW universe with a scale factor $a(t)$.

The initial conditions at the beginning of preheating are determined by the preceding stage of inflation. One can define the moment when preheating starts as the moment when the velocity of the field $\phi$ is zero in conformal time $\eta, d \eta=d t / a(t), a(0)=1$. This happens when $\phi(0) \approx 0.35 M_{\mathrm{Pl}}$ [ [] $]$. We will use rescaled conformal time $\tau=\sqrt{\lambda} \phi(0) \eta$. Inhomogeneous modes of $\phi$ and all modes of $X$ are taken to be in their conformal vacua at $\tau=0$. We consider the case $v \lesssim 10^{-3} M_{\mathrm{Pl}}, g^{2} \gg \lambda$, and take $\lambda=10^{-13}$ [1]. The strength of the resonance depends nonmonotonically on the resonance parameter $q=g^{2} / 4 \lambda$, being maximal around $q=n^{2} / 2[10$. The condition $g^{2} / \lambda \approx 2 n^{2} \gg 1$ means that the evolution begins in the regime of a broad parametric resonance.

The equations of motion for $\phi$ and $X$ are

$$
\begin{aligned}
& \ddot{\phi}+3 H \dot{\phi}-\nabla^{2} \phi / a^{2}+\lambda\left(\phi^{2}-v^{2}\right) \phi+g^{2} X^{2} \phi=0, \\
& \ddot{X}_{i}+3 H \dot{X}_{i}-\nabla^{2} X_{i} / a^{2}+g^{2} \phi^{2} X_{i}=0,
\end{aligned}
$$

where $H=\dot{a} / a$. Substituting $\phi=\phi_{0}+\delta \phi$, where $\phi_{0}$ is the inflaton's homogeneous ("zero") mode, into (2) and assembling terms linear in $\phi_{0}$, we obtain the effective mass of $\phi$ :

$$
m_{\text {eff }}^{2}=-\lambda v^{2}+3 \lambda\left\langle(\delta \phi)^{2}\right\rangle+g^{2}\left\langle X^{2}\right\rangle .
$$

Angular brackets denote spatial averaging. Because fluctuations rapidly become classical 佃, their variances can be computed as spatial averages; thus $\left\langle X^{2}\right\rangle$ is the variance of $X$. The maximal value of $\left\langle X^{2}\right\rangle$ grows with $N$, while that of $\left\langle(\delta \phi)^{2}\right\rangle=\left\langle\left(\phi-\phi_{0}\right)^{2}\right\rangle$ does not 6, 11]. We 
therefore expect that at large $N$ fluctuations of $X$ will play a more important role in (4) than those of $\phi$, at least for some time. In general, there is no guarantee that a useful effective potential can be defined for a state far from thermal equilibrium. We have found that in our case Eq. (4) gives a good working definition for the effective mass.

The idea of nonthermal phase transitions [2] is that large fluctuations of $\left\langle X^{2}\right\rangle$ (and $\left\langle(\delta \phi)^{2}\right\rangle$ ) generated during preheating can change the shape of the effective potential and lead to symmetry restoration. Afterwards, the universe expands, the values of $\left\langle X^{2}\right\rangle$ and $\left\langle(\delta \phi)^{2}\right\rangle$ drop down, and the phase transition with symmetry breaking occurs. In the case $g^{2} / \lambda \gg 1$ the phase transition can be of the first order. This can be expected by analogy with the usual thermal case [14]. Let us establish the necessary conditions for this transition to occur and to be of the first order.

(i) At the time of the phase transition, the point $\phi=$ 0 should be a local minimum of the effective potential. From (4i), we see that this means that $g^{2}\left\langle X^{2}\right\rangle>\lambda v^{2}$.

(ii) At the same time, the typical momentum $p_{*}$ of $X$ particles should be smaller than $g v$. This is the condition of the existence of a potential barrier. Particles with momenta $p<g v$ cannot penetrate the state with $|\phi| \approx v$, so they cannot change the shape of the effective potential at $|\phi| \approx v$. Therefore, if both conditions (i) and (ii) are satisfied, the effective potential has a local minimum at $\phi=0$ and two degenerate minima at $\phi \approx \pm v$.

(iii) Before the minima at $\phi \approx \pm v$ become deeper than the minimum at $\phi=0$, the inflaton's zero mode should decay significantly, so that it performs small oscillations near $\phi=0$. Then, after the minimum at $|\phi| \approx v$ becomes deeper than the minimum at $\phi=0$, fluctuations of $\phi$ drive the system over the potential barrier, creating an expanding bubble.

Before performing numerical investigation of any particular model one may want to find out in which cases these conditions can be satisfied. If the initial value of $\phi_{0}$ is much larger than $v$, the first stages of preheating proceed in the same way as in the conformal theories $(v=0)$, which is a well established case. The maximal value of $\left\langle X^{2}\right\rangle$ is reached at some time $\tau_{\max }$. The previous studies [6,11] indicated that $\left\langle X^{2}\right\rangle_{\max } \sim \sqrt{N} \phi^{2}(0) / q a^{2}\left(\tau_{\max }\right)$. We need, however, an estimate for $\left\langle X^{2}\right\rangle$ at the time of the phase transition. If, at $\tau>\tau_{\max },\left\langle X^{2}\right\rangle$ merely decreased with time due to the redshift, we could estimate it by simply replacing $a\left(\tau_{\max }\right)$ with the current value of $a$. In reality, fluctuations of $X$ decrease somewhat slower, because of the continuing decay of the inflaton, but since the condition (i) holds whenever $v$ is small enough compared to fluctuations, it is safe to make the replacement $a\left(\tau_{\max }\right) \rightarrow a$. As for the condition (ii), we note that the scale of momenta of $X$ particles is set by the frequency of the inflaton's oscillations, $p_{*} \sim \sqrt{\lambda} \phi(0) / a$, although numerical factors are to be expected in this estimate. Both conditions (i) and (ii) are met if $\frac{\phi^{2}(0)}{a^{2} q} \ll v^{2} \ll \sqrt{N} \frac{\phi^{2}(0)}{a^{2}}$.
We see that the window allowed for $v^{2}$ grows with $q$ and with $N$. Therefore we will explore cases $g^{2} / \lambda \gg 1$ and $N \geq 1$.

The condition (iii) cannot be verified using only results of the previous studies. Nevertheless, we may expect a fairly rapid decay of the zero mode by analogy with such a decay in the model with a massive inflaton [6]. The analogy is relevant when the amplitude of $\phi_{0}$ in the present model becomes comparable to $v$, so that deviations from the conformal invariance set in. This expectation is well confirmed by our numerical results.

For numerical studies, the full nonlinear equations of motion (2), (3) were solved directly in the configuration space. The computations were done on $64^{3}$ lattices for $N=1,2,9$ and on $128^{3}$ lattices for $N=1,2$. Below we present results for the model with parameters $g^{2} / \lambda=200$ and $v=0.7 \times 10^{-3} M_{\mathrm{Pl}} \approx 0.8 \times 10^{16} \mathrm{GeV}$, and a twocomponent $X$, obtained on a $128^{3}$ lattice, with the expansion of the universe assumed to be radiation dominated.

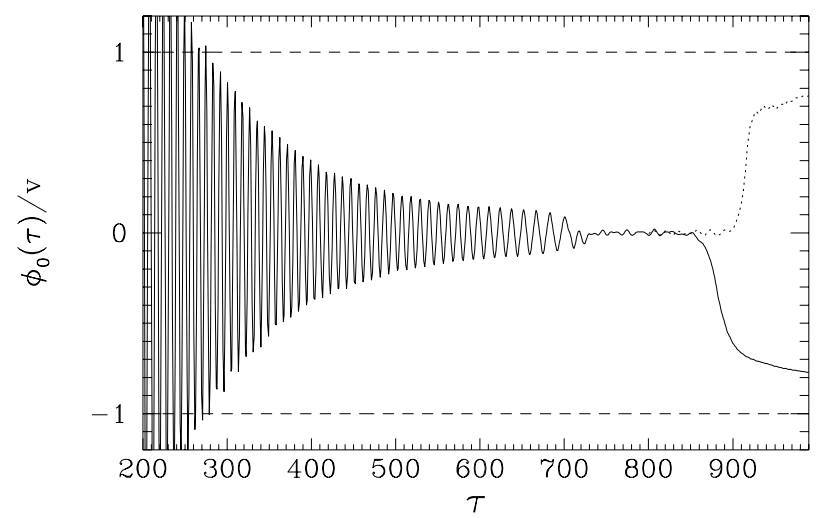

FIG. 1. Time dependence of the zero-momentum mode of $\phi$ in units of its vacuum value for two runs with different realizations of random initial conditions for fluctuations. All other parameters were the same in both runs; $\tau$ is conformal time in units of $\left(\lambda \phi^{2}(0)\right)^{-1 / 2}$.

Time dependence of the zero mode $\phi_{0}$ is shown in Fig. 11. Initially $\phi_{0}$ oscillates with a large amplitude $\bar{\phi} \gg v$. If all fluctuations were absent, the zero mode $\phi_{0}$, in the expanding universe, would soon start oscillations near one of its vacuum values, $\pm v$. This would happen when the amplitude of the oscillations became smaller that $\sqrt{2} v$. In Fig. 1 we see that the actual dynamics is completely different. The zero mode of the field $\phi$ continues to oscillate near $\phi=0$ even when its amplitude becomes much smaller than $v$. In other words, the field oscillates on top of the local maximum of the bare potential. This can occur only because the effective potential acquires a minimum at $\phi=0$ due to interaction of the field $\phi$ with $\left\langle X^{2}\right\rangle$.

At still later times, $\tau \gtrsim 720$ in Fig. 1, the zero mode of $\phi$ decays completely. This should be interpreted as restoration of the symmetry $\phi \rightarrow-\phi$ by nonthermal fluctuations. Finally, at $\tau \gtrsim 860$ a phase transition occurs, 
and the symmetry breaks down.

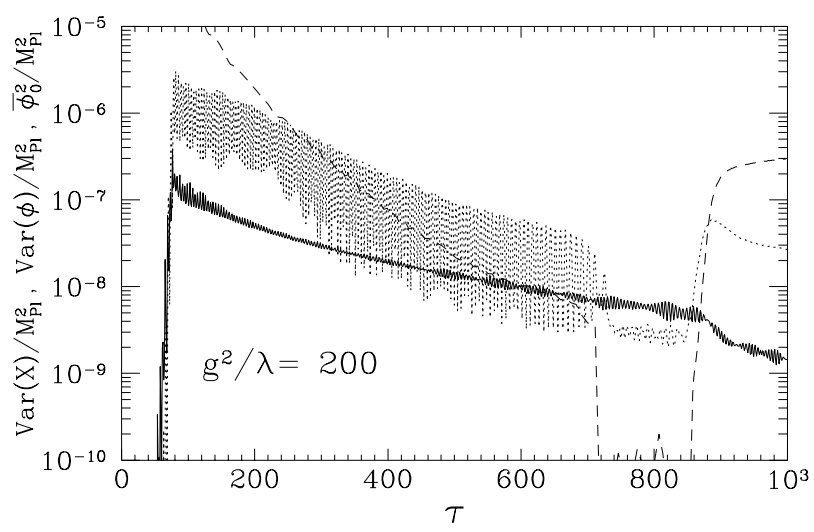

FIG. 2. Variance of $X$ (solid line), variance of $\phi$ (dotted line), and amplitude of the zero-momentum mode of $\phi$ (dashed line) as functions of time.

Variances of the fields $X$ and $\phi$ as functions of time are shown in Fig. 2. One can readily estimate that fluctuations of $X$ (whose variance is multiplied by a larger coupling constant $g^{2}$ ) give a dominant contribution to the effective potential, compared to fluctuations of $\phi$ itself. At the moment of the phase transition $(\tau \approx 860$ in Fig. 2), $g^{2}\left\langle X^{2}\right\rangle$ is still larger than $\lambda v^{2}$. This means that the effective potential still has a local minimum at $\phi=0$ even after the phase transition, which confirms that the transition is of the first order.

To make sure that the phase transition itself is not a lattice artifact, we have varied the size $L$ of the integration box and the number of grid points. Values of $L$ should be chosen in such a way that, on the one hand, there is enough control over the ultraviolet part of the spectrum for the results to be cutoff-independent and, on the other hand, the infrared part of the spectrum is represented well enough, so that the order of the phase transition is determined correctly. Good choices of $L$ are made by monitoring power spectra of the fields, such as those shown in Fig. 3 .

At late times (but prior to the phase transition) the power spectra weakly depend on time and are power law functions at small $k$ with an exponential tail at large $k$ (conformal momentum $k$ is defined in units of $\sqrt{\lambda} \phi(0)$, $k \equiv p a / \sqrt{\lambda} \phi(0))$. We made sure that the exponential tail of the power spectra is resolved in our simulations. Under that condition, the power laws do not significantly change with $L$, the number of grid points and, additionally, with the number of components of $X$. The power law for $X$ is fitted by $P_{X}(k) \propto k^{-2.2}$ for $k>1$. At $\tau \approx 700$, $k \approx 10$ corresponds to physical momentum $p \approx g v$. Later on, this value of the physical momentum corresponds to larger $k$, and at the moment of the phase transition it corresponds to momenta on the exponential tail of the power spectrum, which signifies that the condition (ii) is fulfilled, see Fig. 3. Note the enhancement of power spectra at small $k$ occurring during and after the phase transition. This is a signature of a bubble of the new phase and of the "soft" fluctuations produced in bubble collisions 15 .

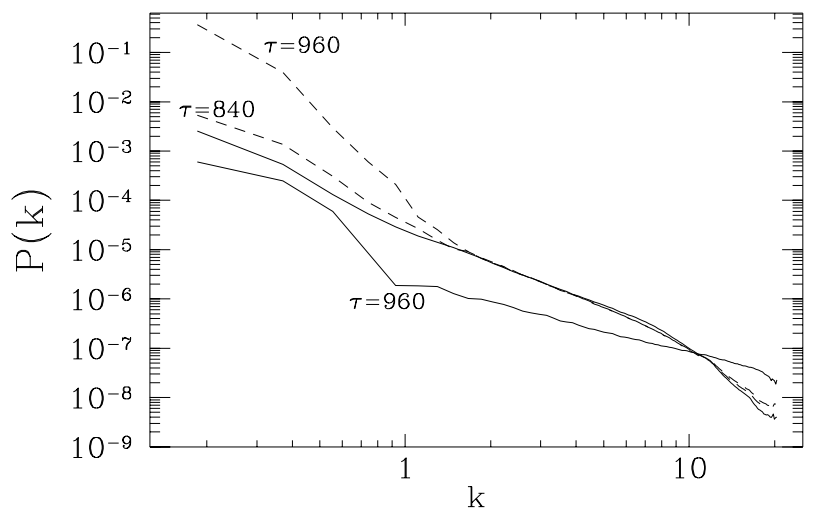

FIG. 3. Power spectra of the fields $X_{1} / a$ (solid line) and $\phi / a$ (dotted line) before and after the phase transition.

We have found that the transition grew stronger when we captured more of the infrared region, either through an increase of the grid size at fixed maximal momentum or through an increase of $L$ itself. Thus we expect that the phase transition will be even more strongly first-order if we use larger lattices for our simulations.

The transition also became more strongly first-order when we increased $g^{2} / \lambda$ or the number $N$ of fields $X_{i}$. Nevertheless, we observed a first-order transition even at $N=1$.

Direct demonstrations of the first-order nature of the transition are obtained by plotting a probability distribution of values of $\phi$ over grid points and the actual field configuration in space. These are shown in Figs. 4, 5 .

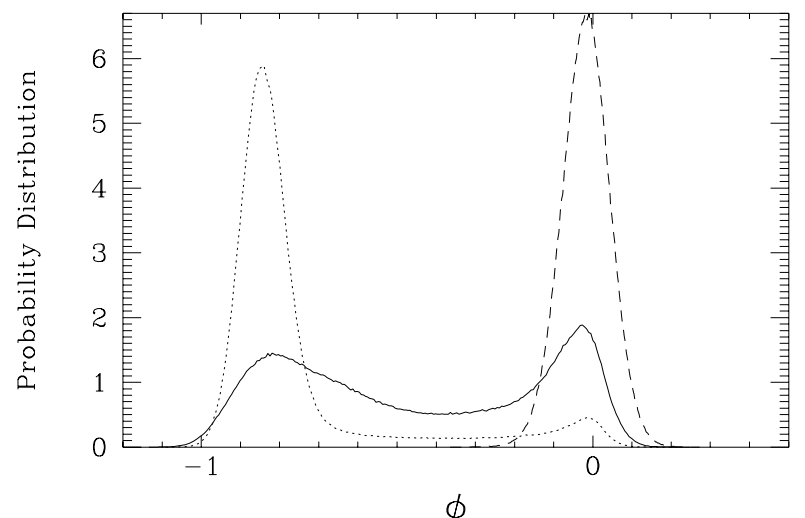

FIG. 4. Probability distribution of $\phi(\mathbf{x})$ at several moments of time just before (dashed line), during (solid line), and at the end of the phase transition (dotted line).

Prior to the phase transition, we observe a single peak in the probability distribution function of $\phi$. The peak is centered at $\phi \approx 0$, although its position oscillates slightly, synchronously with the oscillations of the zero mode. The 
width of the peak is related to the variance $\left\langle(\delta \phi)^{2}\right\rangle$. At the moment of the phase transition, a distinctive second peak appears near the vacuum value of the field (position of this second peak is shifted somewhat from the vacuum value, because fluctuations still give a significant contribution to the effective potential). After that, the second peak grows, while the peak at $\phi=0$ decreases and eventually disappears. During this period, positions of both peaks do not change. For some realizations of random initial conditions for fluctuations, the second peak appeared at $\phi \approx v$; for others, at $\phi \approx-v$. The time of the phase transition was also changing somewhat with the realization. This is an unambiguous signature of spontaneous nucleation of a bubble of the new phase in a volume occupied by the old phase.

By plotting the actual field configuration in space, we have indeed directly observed nucleation of a single bubble and the bubble's subsequent expansion until it was occupying the whole integration volume. The field configuration at the beginning of the phase transition is shown in Fig. 5. To the best of our knowledge, this is the first time when lattice simulations allowed one to see nucleation of bubbles during a first-order phase transition.

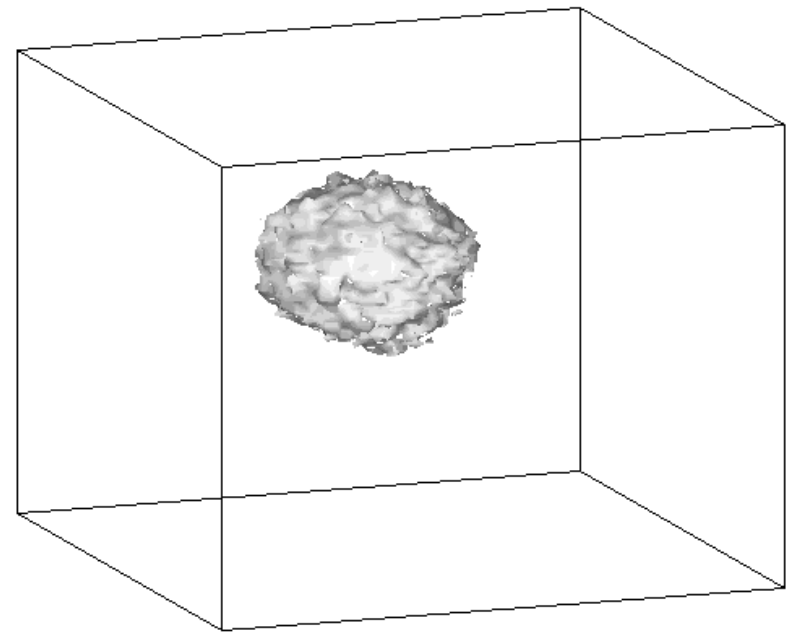

FIG. 5. Bubble of the new phase. We plot a surface of the constant field $\phi=-0.7 v$ at the beginning of the phase transition. Inside the surface $\phi<-0.7 v$.

Let us describe some cosmological implications of our results. Models that exhibit behavior shown in Fig. 1 will lead to domain structure surviving till present. This conclusion is important because it allows us to rule out a large class of cosmological models that lead to domain wall creation. A different behavior of the zero mode is observed at smaller values of $g^{2} / \lambda$ or $N$ (but still $g^{2} / \lambda \gg 1$ ). There, the phase transition occurs when the zero mode still oscillates near $\phi=0$ with a relatively large amplitude. This is a new, specifically nonthermal, type of a phase transition. In such cases, bubbles of $+v$ and $-v$ phases will be nucleated in turn, but their abundances need not be equal, and for certain values of the parameters one of the phases may happen not to form infinite domains. Such models are not ruled out and in fact may have an interesting observable consequence, an enhanced (by bubble wall collisions) background of relic gravitational waves produced by the mechanism proposed in Ref. [7]. In models where the field $\phi$ has many components, the phase transition can lead to creation of strings or monopoles, instead of domain walls 13]. Finally, if the ratio $g^{2} / \lambda$ is sufficiently large, one may expect a short secondary stage of inflation [2]. To investigate this possibility one would need to study models with $g^{2} / \lambda \gg 10^{2}$ or, equivalently, $g^{2} \gg 10^{-11}$, which is quite realistic. However, numerical investigation of this regime requires lattices of a much greater size than we currently use. We hope to return to this issue in a future publication.

This work was supported in part by DOE grant DEFG02-91ER40681 (Task B) (S.K. and I.T.), NSF grants PHY-9219345 (A.L.), PHY-9501458 (S.K. and I.T.), AST95-29-225 (L.K. and A.L.), and by the Alfred P. Sloan Foundation (S.K.).

[1] A. D. Linde, Particle Physics and Inflationary Cosmology (Harwood, Chur, Switzerland, 1990); E. W. Kolb and M. S. Turner, The Early Universe (Addison-Wesley, Redwood City, California, 1990).

[2] L. Kofman, A. Linde, and A. A. Starobinsky, Phys. Rev. Lett. 76, 1011 (1996); I. I. Tkachev, Phys. Lett. B376, 35 (1996).

[3] L. Kofman, A. Linde, and A. A. Starobinsky, Phys. Rev. Lett. 73, 3195 (1994).

[4] S. Khlebnikov and I. I. Tkachev, Phys. Rev. Lett. 77, 219 (1996).

[5] S. Khlebnikov and I. I. Tkachev, Phys. Lett. B390, 80 (1997).

[6] S. Khlebnikov and I. I. Tkachev, Phys. Rev. Lett. 79, 1607 (1997).

[7] S. Khlebnikov and I. I. Tkachev, Phys. Rev. D 56, 653 (1997).

[8] T. Prokopec and T. G. Roos, Phys. Rev. D 55, 3768 (1997); B. R. Greene, T. Prokopec, and T. G. Roos, Phys. Rev. D 56, 6484 (1997).

[9] L. Kofman, A. Linde, and A. A. Starobinsky, Phys. Rev. D 56, 3258 (1997).

[10] P. B. Greene, L. Kofman, A. Linde, and A. A. Starobinsky, Phys. Rev. D 56, 6175 (1997).

[11] S. Khlebnikov, hep-ph/9708313.

[12] D. Boyanovsky, H.J. de Vega, R. Holman, J.F.J. Salgado, Phys. Rev. D 54, 7570 (1996); S. Kasuya and M. Kawasaki, Phys. Rev. D56, 7597 (1997).

[13] I. I. Tkachev, L. Kofman, A. Linde, A. A. Starobinsky and S. Khlebnikov, in preparation.

[14] D.A. Kirzhnits and A.D. Linde, Ann. Phys. (N.Y.) 101, 195 (1976).

[15] E. W. Kolb, A. Riotto and I. I. Tkachev, Phys. Rev. D D56, 6133 (1997). 